\title{
Upregulated miR-20a-5p expression promotes proliferation and invasion of head and neck squamous cell carcinoma cells by targeting of TNFRSF21
}

\author{
HONG WU ${ }^{1,2}$, PAI PANG ${ }^{1}$, MIN-DA LIU ${ }^{1}$, SONG WANG ${ }^{1}$, SHAN JIN $^{1}$, FA-YU LIU ${ }^{1}$ and CHANG-FU SUN ${ }^{1}$ \\ ${ }^{1}$ Department of Oromaxillofacial-Head and Neck Surgery, Oral Maxillofacial Surgery, \\ School of Stomatology, China Medical University, Shenyang, Liaoning 110002; ${ }^{2}$ School of Stomatology, \\ Shenyang Medical College, Shenyang, Liaoning 110034, P.R. China
}

Received November 18, 2017; Accepted May 22, 2018

DOI: 10.3892/or.2018.6477

\begin{abstract}
MicroRNAs (miRNAs) play important roles in regulation of proliferation, migration, and invasion of head and neck squamous cell carcinoma (HNSCC). The present study assessed expression, functions and mechanisms of miR-20a-5p in the regulation of HNSCC cell proliferation, migration and invasion. miR-20a-5p expression in HNSCC cell lines and tissues was detected using qRT-PCR, while miR-20a-5p mimics and inhibitor were transfected into HNSCC cells for assessment of the effects using different assays (CCK-8, wound healing and Transwell assays) and expression of miR-20a-5p-targeting genes (using western blot and luciferase reporter assays). The data revealed that miR-20a-5p was upregulated in both HNSCC tissues and metastatic HNSCC cells. Upregulated miR-20a-5p expression in HNSCC cells promoted tumor cell proliferation, migration and invasion capacities, but resulted in downregulation of TNFRSF21 expression and in turn upregulation of C-C motif chemokine receptor 7 (CCR7) in HNSCC cells. Concordantly, knockdown of miR-20a-5p in HNSCC had the opposite results. In conclusion, miR-20a-5p functioned as an oncogene in HNSCC by downregulating TNFRSF21 and subsequently, upregulating CCR7 expression.
\end{abstract}

Correspondence to: $\mathrm{Dr} \mathrm{Fa}-\mathrm{Yu} \mathrm{Liu}$ or $\mathrm{Dr}$ Chang-Fu Sun, Department of Oromaxillofacial-Head and Neck Surgery, Oral Maxillofacial Surgery, School of Stomatology, China Medical University, 117 Nanjing North Street, Heping, Shenyang, Liaoning 110002, P.R. China

E-mail:1fyhjk@126.com

E-mail: changfusun@hotmail.com

Key words: miR-20a-5p, head and neck squamous cell carcinoma, TNFRSF21, CCR7

\section{Introduction}

Head and neck cancer is still a significant health problem in the world that seriously affects the quality of life of patients. Histologically, the most common form of head and neck cancer is squamous cell carcinoma (HNSCC). HNSCC occurs in the oral and nasal cavity, paranasal sinuses, salivary glands, pharynx and larynx, and HNSCC risk factors include chewing or smoking tobacco, heavy alcohol consumption, and infection of Epstein-Barr virus (EBV) or human papilloma virus (HPV) $(1,2)$. Head and neck cancer is the sixth most commonly diagnosed malignancy in the world with $>500,000$ new cancer cases annually (1), and the overall 5 -year survival rate is low due to tumor local recurrence and regional lymph node metastasis (2). Early detection and prediction of prognosis could help medical oncologists to effectively manage this deadly disease.

MicroRNAs (miRNAs) are a class of non-coding small RNAs of $\sim 18-25$ nucleotides in length. miRNAs function to post-transcriptionally regulate gene expression by degradation and/or inhibition of the target mRNA and its translation $(3,4)$. Thus, miRNAs play important roles in the human body, during events such as embryo development, stem cell differentiation, and cell growth and apoptosis, and altered expression of a given miRNA could result in disease development and progression, including human cancers $(3,4)$. Early studies have revealed that aberrant expression of different miRNAs was associated with HNSCC development and progression (5-12). Altered levels of particular miRNAs were assessed as diagnostic markers for HNSCC metastasis (9), while another study revealed that circulating miRNAs could serve as biomarkers to assess the efficacy of therapy and the prognosis of HNSCC (10). miRNAs were also researched as molecular targets or biomarkers in HNSCC therapy (13).

In this study, we focused on miR-20a-5p, which has been reported to be associated with radioresistance of HNSCC cells (14) and to promote proliferation and migration of human hepatocellular and colorectal cancer cells $(15,16)$. We assessed the expression, function and mechanism of action of miR-20a-5p in HNSCC and its role in the regulation of HNSCC cell proliferation, migration and invasion in vitro. 


\section{Materials and methods}

Cell lines and culture. HNSCC cell lines PCI-37A and PCI-37B were generated from one HNSCC patient (37A was derived from a primary tumor, and 37B was derived from lymph node metastasis of the same HNSCC patient). These cell lines were characterized in the Cancer Institute of Pittsburgh University (Pittsburgh, PA, USA) and cultured in low glucose Dulbecco's modified Eagle's medium (DMEM; HyClone Laboratories; Thermo Fisher Scientific, Inc., Logan, UT, USA) supplemented with $10 \%$ fetal bovine serum (FBS; Gibco-BRL; Thermo Fisher Scientific, Inc., Gaithersburg, MD, USA), 100 U/ml penicillin (Sigma-Aldrich Chemical Co., St. Louis, MO, USA) and $100 \mu \mathrm{g} / \mathrm{ml}$ streptomycin (Sigma-Aldrich) in a humidified incubator with $5 \% \mathrm{CO}_{2}$ at $37^{\circ} \mathrm{C}$.

Tissue specimens. Surgical tissue specimens were obtained from 15 HNSCC patients at the Department of Oromaxillofacial-Head and Neck Surgery, School of Stomatology (Shenyang China Medical University between August 2014 and September 2014. The present study was approved by the Ethics Committee of China Medical University and all the patients signed an informed consent form before being enrolled in this study. None of the patients had received any chemotherapy or radiotherapy prior to surgery, and all tissue specimens were obtained from surgical resection of the primary tumors, along with paired samples of adjacent normal tissues. The tissues were snap-frozen in liquid nitrogen and stored at $-80^{\circ} \mathrm{C}$. Histological diagnosis was performed and confirmed by the Department of Pathology, School of Stomatology, China Medical University, and the clinicopathological data are displayed in Table I.

RNA isolation and real-time polymerase chain reaction $(R T-P C R)$. Total RNA from cell lines and tissue samples was isolated using TRIzol reagent (Takara Biotechnology, Co., Ltd., Dalian, China) and subsequently reverse-transcribed into cDNA using the Super M-MLV reverse-transcription kit (BioTek, Beijing, China) according to the manufacturers' instructions. qPCR amplification was then conducted using the ABI PRISM 7500 Sequence Detection System (ABI7500; Applied Biosystems; Thermo Fisher Scientific, Inc., Foster City, CA, USA) with the following conditions: $95^{\circ} \mathrm{C}$ for $30 \mathrm{sec}$, then $95^{\circ} \mathrm{C}$ for $5 \mathrm{sec}$, and $60^{\circ} \mathrm{C}$ for $30-34 \mathrm{sec}$ for 40 cycles. The relative miR-20a-5p expression was normalized to U6 expression levels. The qPCR primers for miR-20a-5p were: 5'-CGA CAGTGACCGATTTCTCCT-3' and 5'-GTGCAGGGTCCG AGGTATTC-3'; while the U6 primers were: 5'-CTCGCTTCG GCAGCACA-3' and 5'-AACGCTTCACGAATTTGCGT-3'. The relative expression level of miR-20a-5p was determined using the $2^{-\Delta \Delta \mathrm{Ct}}$ analytical method. In the analysis of the tissue specimens, a calibration sample was used to ensure all reactions occurred in similar conditions. All experiments were carried out in triplicate.

Transient transfection. miR-20a-5p mimics and inhibitor were obtained from GenePharma (Shanghai, China), and transiently transfected into HNSCC cells using Lipofectamine 2000 (Invitrogen; Thermo Fisher Scientific, Inc., Carlsbad, CA, USA) according to the manufacturer's instructions. The cells were subjected to different assays thereafter.

CCK-8 assay. The Cell Counting Kit-8 (CCK-8) assay kit (Beyotime Institute of Biotechnology, Shanghai, China) was used to evaluate tumor cell viability. In brief, cells were seeded into 96-well plates at a density of $\sim 3,000$ cells/well and cultured for $24 \mathrm{~h}$ before transfection with miR-20a-5p mimics or the inhibitor. The cells were then further cultured for 0,24 , 48, 72 and $96 \mathrm{~h}$, respectively. At the end of each experiment, the culture medium was replaced with $100 \mu 1$ fresh serum-free DMEM plus $10 \mu$ l CCK-8 per well, and then the cells were incubated at $37^{\circ} \mathrm{C}$ for an additional $1 \mathrm{~h}$. The optical density value at $450 \mathrm{~nm}$ (OD450) was measured using a plate reader (model ELX-800; BioTek Instruments, Inc., Winooski, VT, USA). All experiments were carried out in triplicate and repeated at least thrice.

Wound healing assay. HNSCC cells were seeded at a density of $3 \times 10^{5}$ cells/well in 6-well culture plates, incubated for $24 \mathrm{~h}$, and then transfected with miR-20a-5p mimics or the inhibitor. After the cells had formed a monolayer, scratches were made on the cell monolayer with a sterile $200-\mu 1$ pipette tip. The cells were washed twice with phosphate-buffered saline (PBS; HyClone; Thermo Fisher Scientific, Inc.) and further incubated in serum-free medium. The wound area was photographed at 0,12 and $24 \mathrm{~h}$ under an inverted phase-contrast microscope. The cell migration distance was then estimated using software. All experiments were carried out in triplicate and repeated at least once.

Transwell migration and invasion assays. Cell migration and invasion capacity was assessed using Transwell chambers (Corning Inc., Corning, NY, USA). In the migration experiment, the Transwell chamber filters were not coated with Matrigel (BD Biosciences, San Jose, CA, USA), however they were coated with $500 \mu \mathrm{l}$ of $30 \%$ Matrigel for the invasion assay. Briefly, the Transwell chambers were placed into a 24-well plate, and $800 \mu$ l DMEM containing 20\% FBS was added to the lower chambers. The corresponding upper chambers were seeded with $1 \times 10^{4}$ transfected cells in $200 \mu 1$ DMEM without FBS. The plates were then cultured for $24 \mathrm{~h}$, and at the end of the experiments, the cells on the surface of the upper chamber were removed using a cotton swab and washed with PBS twice. The cells that had migrated to the bottom side of the filters were fixed in $4 \%$ paraformaldehyde for $20 \mathrm{~min}$ at room temperature and then stained with $0.5 \%$ crystal violet (Amresco, LLC, Solon, OH, USA) for $5 \mathrm{~min}$. The filters were then reviewed, and the number of cells was counted under an inverted phase-contrast microscope (magnification, x200) in five random fields/Transwell chamber.

Western blot analysis. A C-C motif chemokine receptor 7 (CCR7)-specific monoclonal antibody (mouse anti-human CCR7 antibody) and a rabbit anti-TNFRSF21 antibody were purchased from Boyan Technology Co., Ltd. (Shanghai, China; cat. no. BYK-1305R) and Abnova (Walnut, CA, USA; cat. no. PAB0156), respectively, while a monoclonal anti- $\beta$-actin antibody (Wanleibio, Shenyang, China; cat. no. WL01845;) was obtained from Sigma-Aldrich 
Table I. Clinicopathological data of patients.

\begin{tabular}{cccllccc}
\hline $\begin{array}{l}\text { Patient } \\
\text { no. }\end{array}$ & $\begin{array}{c}\text { Age } \\
\text { (years) }\end{array}$ & Sex & $\begin{array}{c}\text { Primary tumor } \\
\text { site }\end{array}$ & $\begin{array}{c}\text { Tumor } \\
\text { differentiation }\end{array}$ & $\begin{array}{c}\text { Lymph node } \\
\text { metastasis }\end{array}$ & TNM & Clinical stage \\
\hline 1 & 59 & Male & Bucca & Moderate & N & T2N0M0 & II \\
2 & 54 & Male & Soft palate & High & N & T2N0M0 & II \\
3 & 50 & Male & Tongue & Moderate & Y & T2N1M0 & III \\
4 & 51 & Male & Mouth floor & Poor & N & T2N0M0 & II \\
5 & 47 & Male & Soft palate & Moderate & N & T1N0M0 & II \\
6 & 68 & Male & Tongue & Moderate & N & T2N0M0 & II \\
7 & 61 & Male & Gingiva & Moderate & Y & T1N2bM0 & IVA \\
8 & 72 & Male & Tongue & Poor & N & T2N0M0 & II \\
9 & 63 & Male & Tongue & Poor & Y & T2N2cM0 & IVA \\
10 & 58 & Male & Tongue & Moderate & N & T2N0M0 & II \\
11 & 50 & Male & Tongue & Moderate & N & T2N0M0 & II \\
12 & 64 & Female & Bucca & Moderate & N & T2N0M0 & II \\
13 & 66 & Female & Bucca & Moderate & Y & T4N1M0 & IVA \\
14 & 54 & Female & Tongue & Moderate & N & T2N0M0 & II \\
15 & 61 & Female & Tongue & Poor & N & T2N0M0 & II \\
\hline
\end{tabular}

TNM, tumor-node-metastasis.

and used as an internal control. Cells were transfected with miR-20a-5p mimics, inhibitor, or miRNA control for $48 \mathrm{~h}$, washed in ice-cold PBS, and lysed on a plate with ice-cold cell lysis buffer (Wanleibio, Shenyang, China). Cells were then centrifuged for $10,000 \mathrm{x}$ g at $4^{\circ} \mathrm{C}$ for $10 \mathrm{~min}$. The protein concentration was assayed using the BCA assay (Wanleibio), and 40- $\mu \mathrm{g}$ each of these protein samples were fractionated on a $10 \%$ sodium dodecyl sulfate-polyacrylamide gel electrophoresis (SDS-PAGE) gel and transferred onto polyvinylidene difluoride membranes (EMD Millipore, Billerica, MA, USA). For western blotting, the membranes were blocked in 5\% skim milk solution for $1 \mathrm{~h}$ and incubated with the TNFRSF 21 and CCR7 primary antibody (1:100) and the anti- $\beta$-actin antibody $(1: 1,000)$ overnight at $4^{\circ} \mathrm{C}$. The next day the membranes were washed with PBS-Tween-20 (PBST) thrice and then further incubated with horseradish peroxidase-conjugated sheep anti-rabbit $\operatorname{IgG}$ (cat. no. WLA023; Wanleibio) at a dilution of 1:5,000. The protein bands were subsequently visualized by enhanced chemiluminescence (Wanleibio) and quantified using Gel-Pro-Analyzer software (Media Cybernetics, Rockville, MD, USA).

Luciferase reporter assay. Luciferase reporter assays were carried out with a Luciferase detection kit (Promega, Madison, WI, USA). The wild-type (WT) and mutated type (MT) 3'-untranslational regions of TNFRSF21 cDNA were cloned into the pmirGLO reporter luciferase vector. For the luciferase reporter assay, PCI-37B cells were co-transfected with $25 \mathrm{pM}$ miR-20a-5p mimic or negative control (NC) and $0.5 \mu \mathrm{g}$ of the pmirGLO-3'-UTR WT or MT plasmid using Lipofectamine 2000 (Invitrogen; Thermo Fisher Scientific, Inc.). Luciferase activity was then assessed $48 \mathrm{~h}$ after transfection using the Dual-Luciferase Reporter Assay system
(Promega). The experiments were conducted in triplicate and repeated three times.

Statistical analysis. All data analyses were performed using SPSS 13.0 statistical software (SPSS, Inc., Chicago, IL, USA), and the $\chi^{2}$ test and Student's t-test were used to compare two groups of data. A P-value of $<0.05$ was considered to indicate a statistically significant result.

\section{Results}

Upregulated miR-20a-5p levels in HNSCC cell lines and tissues. Our qRT-PCR data revealed that miR-20a-5p expression was higher in 12 out of 15 paired tumor specimens $(80 \%)$ than in the adjacent normal tissues $(\mathrm{P}<0.05)$. miR-20a-5p expression was lower in two tumor specimens (13\%) than in the paired adjacent normal tissues $(\mathrm{P}<0.05)$, and one specimen pair (7\%) exhibited no significant difference in miR-20a-5p expression ( $\mathrm{P}>0.05$; Fig. 1A). In HNSCC cell lines, miR-20a-5p expression was 2.5 times higher in PCI-37B cells than in PCI-37A cells (Fig. 1B).

Expression of miR-20a-5p promotes HNSCC cell proliferation. We then assessed the effects of miR-20a-5p mimics and an inhibitor on HNSCC cell viability, migration and mechanism of action. We transiently transfected PCI-37B cells with miR-20a-5p plasmids and found that miR-20a-5p expression was increased by 2.3 -fold in the mimics group, whereas miR-20a-5p expression was reduced by 0.6 -fold in the inhibitor group (Fig. 2A).

The tumor cell viability CCK- 8 assay revealed that the number of cells in the mimics group was greater than that in the PCI-37B parental and mimics-NC groups, whereas 
A

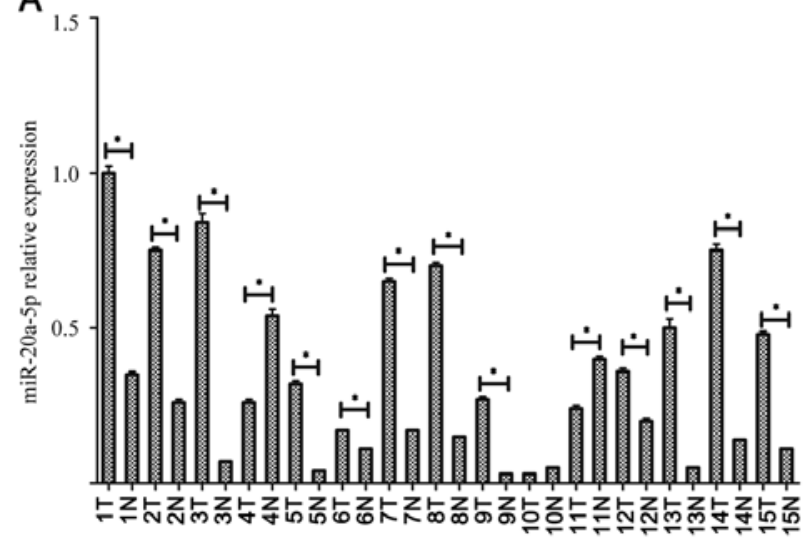

B

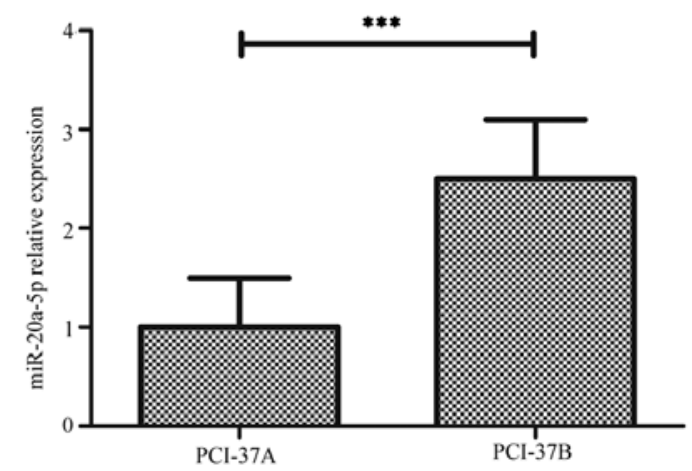

Figure 1. Differential miR-20a-5p expression in head and neck squamous cell carcinoma HNSCC tissues and cells. qRT-PCR was performed to assess the levels of miR-20a-5p in HNSCC cells and tissue specimens vs. paired adjacent normal tissues. ${ }^{*} \mathrm{P}<0.05$ and ${ }^{* * * *} \mathrm{P}<0.001$, respectively. (A) Tissues and (B) cell lines. HNSCC, head and neck squamous cell carcinoma.

A

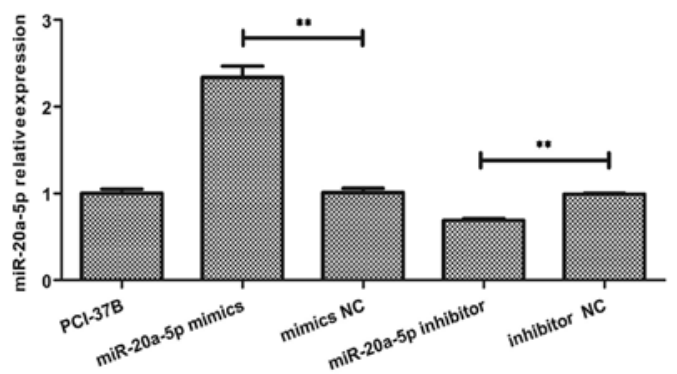

B

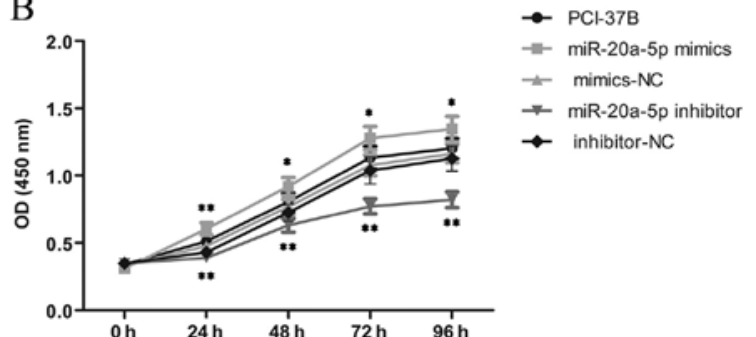

Figure 2. Effects of miR-20a-5p mimics and inhibitor vs. negative controls on the regulation of miR-20a-5p expression and HNSCC PCI-37B cell viability. (A) Transient transfection with miR-20a-5p mimics, inhibitor or miRNA control. ${ }^{* *} \mathrm{P}<0.01$. (B) Cell viability as determined by CCK-8 assay. PCI-37B cells were grown and transiently transfected with miR-20a-5p mimics, inhibitor or miRNA control and subjected to a CCK- 8 assay. ${ }^{*} \mathrm{P}<0.05$ compared with the mimics-NC group, ${ }^{* *} \mathrm{P}<0.01$ compared with the inhibitor-NC group. HNSCC, head and neck squamous cell carcinoma; NC, negative control.

the number of cells in the inhibitor group was less than that in PCI-37B parental and inhibitor-NC groups (Fig. 2B), indicating that miR-20a-5p promoted PCI-37B cell proliferation.

Expression of miR-20a-5p promotes HNSCC cell migration. We next performed tumor cell wound healing and Transwell assays and found that the wound width was narrower in the miR-20a-5p mimics group than in the PCI-37B parental and mimics-NC groups after 12 and $24 \mathrm{~h}$ in culture $(\mathrm{P}<0.05$; Fig. $3 \mathrm{~A})$. In contrast, the wound width was wider in the miR-20a-5p inhibitor group than in the PCI-37B parental and inhibitor-NC groups ( $\mathrm{P}<0.01$; Fig. 3A). Similarly, the Transwell migration assay further confirmed this finding ( $(\mathrm{P}<0.01$; Fig. $3 \mathrm{~B})$.

Expression of miR-20a-5p promotes HNSCC cell invasion. The Transwell invasion assay revealed that compared with that in the control group, upregulation of miR-20a-5p expression using the mimics significantly increased the number of cells that invaded through the Matrigel-coated membrane into the lower chamber, whereas downregulation of miR-20a-5p expression led to the opposite result $(\mathrm{P}<0.01 ;$ Fig. 4).

miR-20a-5p targets TNFRSF21 in HNSCC cells. To explore the mechanism of miR-20a-5p action in HNSCC, we used algorithms (TargetScan, PicTar and miRanda) to predict potential downstream targets of miR-20a-5p and found that TNFRSF21 could be one of the miR-20a-5p targets (miR-20a-5p had 8-mer nucleotides that matched between the TNFRSF21 3'-UTR and miR-20a-5p, which was predicted by TargetScan, miRDB and miRanda programs; Fig. 5A). We therefore conducted luciferase reporter and western blot assays. Our data revealed that luciferase activity was downregulated by $\sim 40 \%$ in the WT group transfected with miR-20a-5p mimics compared with that in the control group $(\mathrm{P}<0.05$; Fig. 5B). However, there was no obvious alteration of luciferase activity in the MT group $(\mathrm{P}>0.05$; Fig. 5B). Moreover, the western blotting results revealed that upregulation of miR-20a-5p expression reduced the level of TNFRSF21 protein in PCI-37B cells by 0.53 -fold compared with expression in the control group $(\mathrm{P}<0.05)$, whereas downregulation of miR-20a-5p expression increased the level of TNFRSF21 protein in PCI-37B cells by 1.6 -fold compared with that in the control group $(\mathrm{P}<0.05$; Fig. $5 \mathrm{C}$ and $\mathrm{D})$. These data revealed that TNFRSF21 is a direct downstream target of miR-20a-5p.

miR-20a-5p upregulates CCR7 level in HNSCC cells. Our previous data revealed the role of CCR7 in HNSCC metastasis by demonstrating that CCR7 expression promoted HNSCC cell migration and invasion through several signaling pathways (17-21). In this study, we also found that miR-20a-5p mimics promoted HNSCC cell migration and invasion (Figs. 3 and 4). We hypothesized that miR-20a-5p may be upstream of signaling in this CCR-7-related tumor cell 
A
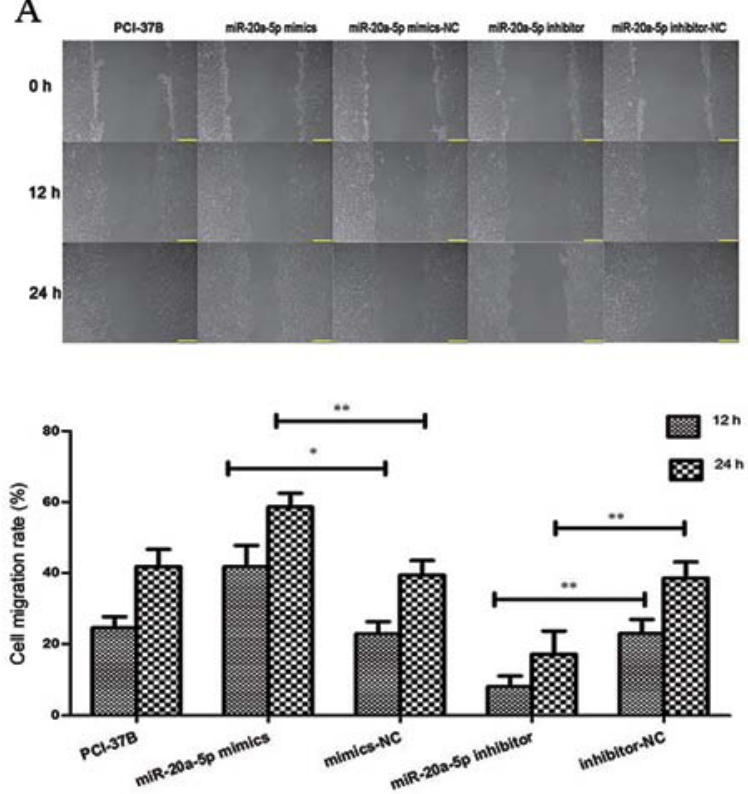

B
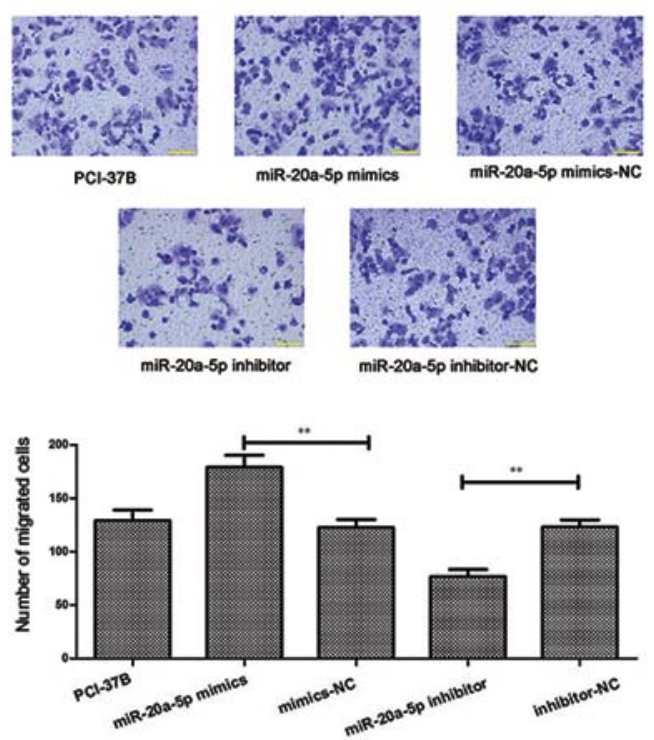

Figure 3. Effects of miR-20a-5p mimics and inhibitor vs. negative controls on the regulation of PCI-37B cell migration. (A) Wound healing assay. PCI-37B cells were grown and transiently transfected with miR-20a-5p mimics, inhibitor, or miRNA control and subjected to a wound healing assay. The graph displays quantified data from the wound healing assay. (B) Transwell migration assay. PCI-37B cells were grown and transiently transfected with miR-20a-5p mimics, inhibitor, or miRNA control and subjected to a Transwell migration assay. ${ }^{*} \mathrm{P}<0.05 ;{ }^{* *} \mathrm{P}<0.01$.

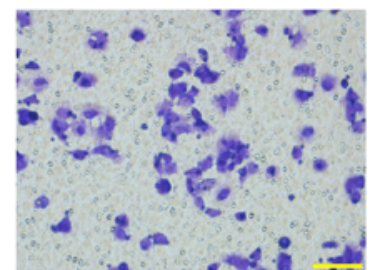

PCl-37B

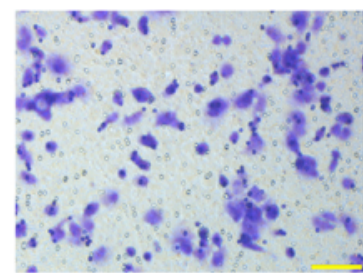

miR-20a-5p mimics

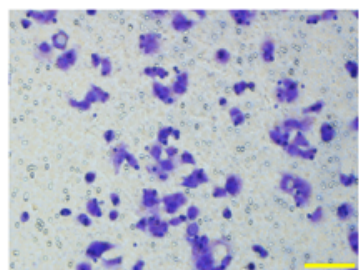

miR-20a-5p mimics-NC

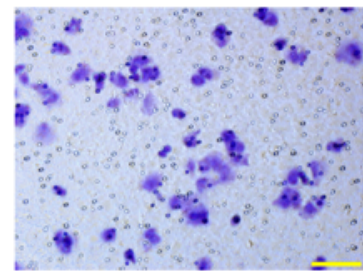

miR-20a-5p inhibitor

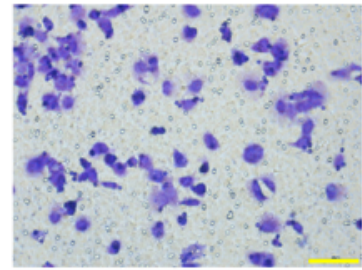

miR-20a-5p inhibitor-NC

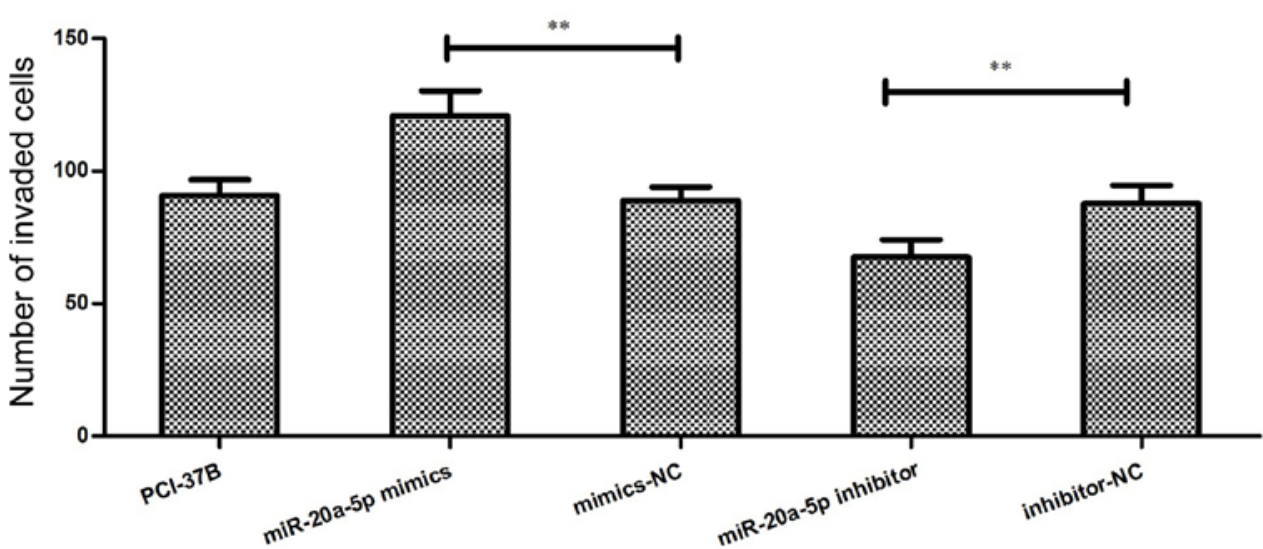

Figure 4. Effects of miR-20a-5p mimics and inhibitor vs. negative controls on the regulation of PCI-37B cell invasion capacity. PCI-37B cells were grown and transiently transfected with miR-20a-5p mimics, inhibitor or miRNA control and subjected to Transwell tumor cell invasion assay. The graph displays the quantified data. ${ }^{* *} \mathrm{P}<0.01$. 

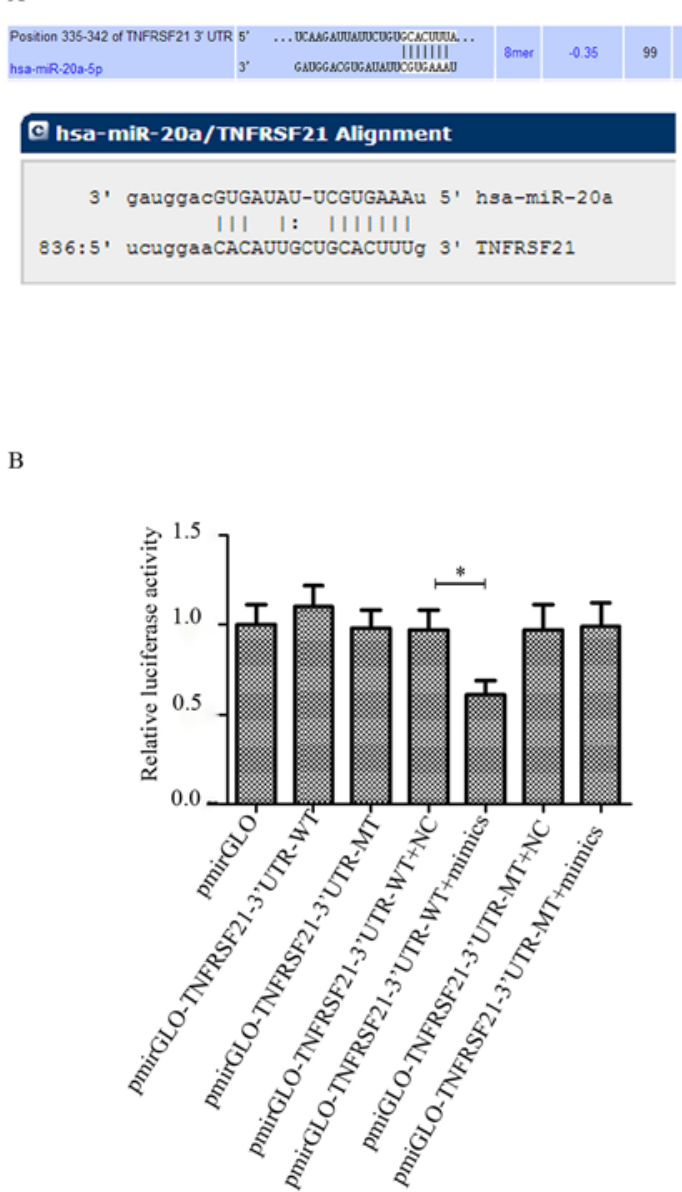

$\mathrm{C}$
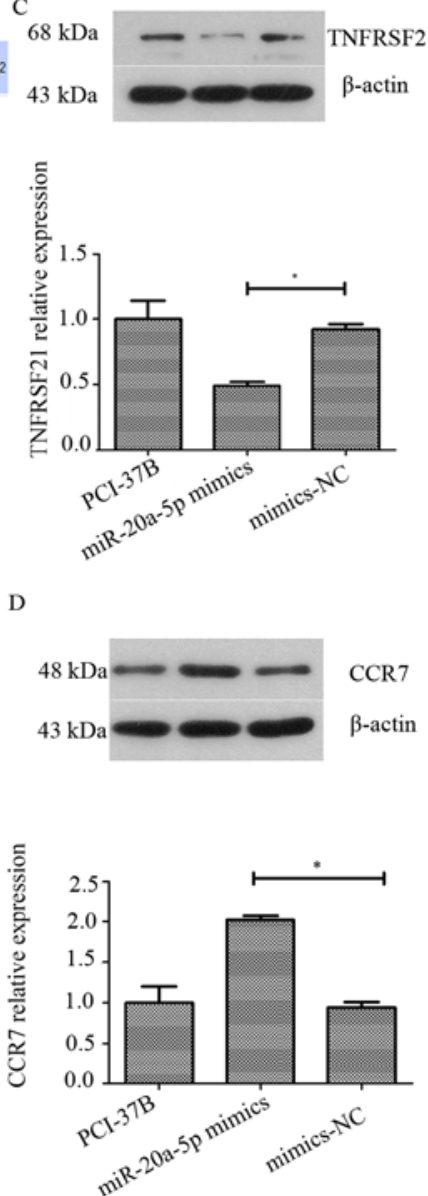
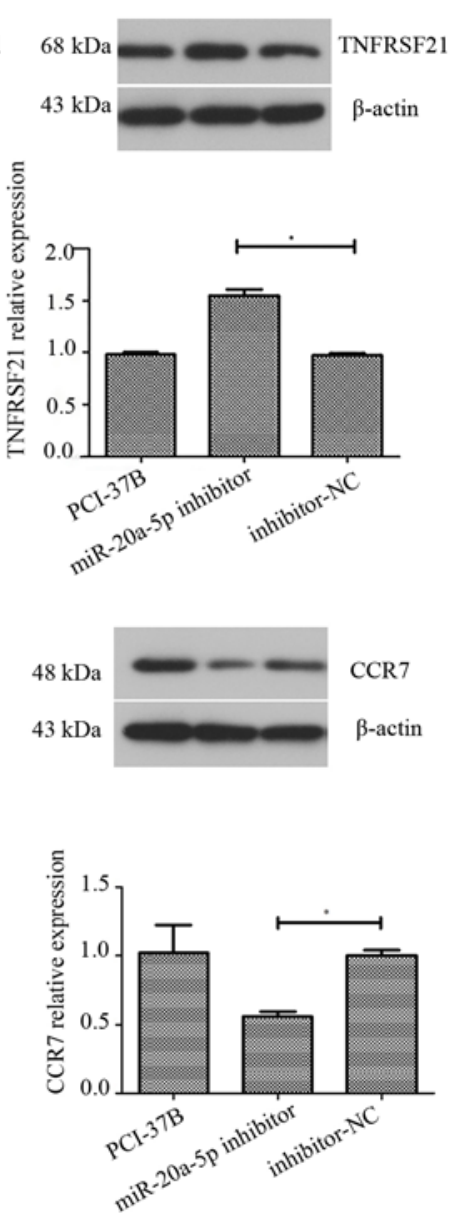

Figure 5. miR-20a-5p mimics targeting TNFRSF21 in PCI-37B cells. (A) Putative binding site of miR-20a-5p to the TNFRSF21 3'-untranslated region. The mutation results in a change from ACT to TGA in the sequence. (B) Luciferase reporter assay. PCI-37B cells were grown and transiently transfected with pmirGLO carrying WT or mutant TNFRSF21 3'-UTR cDNA and miR-20a-5p mimics plasmid and then subjected to Luciferase reporter assay. ${ }^{*}$ P $<0.05$. (C) Western blotting. After transfection with miR-20a-5p mimics or inhibitor and miRNA control for $4 \mathrm{~h}$ (upper panel), the cells were subjected to western blot analysis of TNFRSF21 protein. The graph displays data quantified using Gel-Pro-Analyzer software. *P<0.05. (D) Western blotting. CCR7 expression in PCI-37B cells upon transfection with miR-20a-5p mimics, inhibitor or miRNA control was detected by western blotting. The graph displays data quantified using Gel-Pro-Analyzer software. ${ }^{*} \mathrm{P}<0.05$.

migration and invasion event. Although all three computer databases did not support the notion that miR-20a-5p is able to directly target CCR7, our western blot data revealed that upregulation of miR-20a-5p expression did increase the level of CCR7 protein in PCI-37B cells by 2.15 -fold compared with that of the control group $\mathrm{P}<0.05$ ); however, downregulation of miR-20a-5p expression reduced the level of CCR7 protein in PCI-37B cells by 0.56-fold compared with that of the control group $(\mathrm{P}<0.05$; Fig. 5C), indicating that an indirect effect of miR-20a-5p on CCR-7 expression in HNSCC cells in vitro.

\section{Discussion}

To date, the role of miR-20a-5p in HNSCC remains to be defined, although a number of different studies has revealed aberrant miR-20a-5p expression in a variety of diseases and cancers, including ovarian (22), cervical (23) and gastric cancers (24). Altered miR-20a-5p expression acts as an oncogene to promote tumor cell proliferation and invasion in these cancers, but in skin squamous cell carcinoma (25), thyroid cancer (26), and oral squamous cell carcinoma $(27,28)$, miR-20a-5p was revealed to function as a tumor suppressor gene to inhibit tumor cancer cell proliferation and invasion, indicating that there are organ sites- or target-dependent effects of miR-20a-5p in human cancers. In the present study, we demonstrated miR-20a-5p overexpression in both HNSCC tissues and metastatic HNSCC cells, which let us speculate that miR-20a-5p could be an oncogene in HNSCC. Our further experiments demonstrated that upregulation of miR-20a-5p expression promoted HNSCC cell proliferation, migration and invasion capacity in vitro, whereas downregulation of miR-20a-5p expression inhibited HNSCC cell proliferation, migration and invasion capacity in vitro. A previous study reported that miR-20a-5p expression induced resistance of nasopharyngeal cancer cells to radiation therapy (14), while another study revealed that miR-20a-5p increased the capacity of colorectal cancer invasion and metastasis (16). Our present study further supported these studies, although controversial data do exist; for example, Chang et al (27) profiled differentially expressed miRNAs in oral SCC cell lines and found that the miR-17-92 cluster (miR-17, miR-19b, miR-20a and miR-92a) was significantly downregulated in oral SCC cells (particularly, miR-17 and miR-20a), loss of which was associated with advanced oral squamous cancer 
tumor-node-metastasis (TNM) stage and lymph node metastasis. However, Hu et al (28) linked miR-20a expression to HPV16 E7, a risk factor for HNSCC, and revealed that miR-20a expression was significantly higher in oral SCC tissues compared to adjacent non-tumor tissues and that silencing of HPV-16 E7 expression downregulated the level of miR-20a in tumor cells and, in turn, reduced tumor cell proliferation, invasion and metastasis. Yu et al (29) revealed that when cytokine secretion in the cell microenvironment was changed, the role of miR-20a-5p in breast cancer cells was also altered. A given miRNA was able to play a different role in different cells by targeting and inhibiting expression of different genes $(30,31)$. All of these data indicated that the role of miR-20a-5p in the development of various human cancers remains to be defined and thus, further studies with a larger sample size and more HNSCC cell lines are warranted to confirm our data.

By targeting the expression of a particular gene, miRNAs exert their biological effects on cells; thus, miRNA involvement in different targeting genes could have different roles in human cancers or diseases. In our present study, we investigated the potential genes targeted by miR-20a-5p in HNSCC cells and our bioinformatic analysis revealed that TNFRSF21 could be a potential target of miR-20a-5p, and our luciferase reporter assay confirmed this bioinformatic prediction, revealing that miR-20a-5p bound to the TNFRSF21 3'-UTR, but not to the mutant one. Our western blot results revealed that TNFRSF21 expression was inversely correlated with miR-20a-5p expression, while CCR7 expression was positively correlated with miR-20a-5p expression, which clearly suggest that miR-20a-5p indirectly affected CCR7 expression. This is because a given miRNA could inhibit the expression of the targeting gene, but will not upregulate the expression of the targeting genes. However, previous studies have revealed that miR-20a-5p could directly target different genes in different human cancers, such as TNKS2, EGR2 and LIMK1, to manipulate tumor cell phenotypes and gene expression (23-25), which could help to explain why miR-20a-5p possesses different roles in different human cancers as an oncogene or tumor-suppressor gene. In our present study, miR-20a-5p was able to directly target and regulate TNFRSF21 expression in HNSCC cells.

TNFRSF21, also called death receptor 6 (DR6), is a member of the tumor necrosis factor receptor superfamily (TNFRSF) $(32,33)$. TNFRSF21 is widely expressed in all types of tissues and various cultured cells (32) and after binding to the corresponding ligands, TNFRSF21 is able to induce cell apoptosis $(34,35)$. However, a previous study revealed that negative lymphocytic HLA-DR6 was associated with a favorable 5-year survival of HNSCC patients (36). However, since we did not find a qualified anti-TNFRSF21 antibody commercially available for immunohistochemical analysis of TNFRF21 expression in tumor tissues, we will leave such a project for our future study by collecting fresh tissue samples for western blot analysis of TNFRSF21 expression. Moreover, CCR7 is a member of the G protein-coupled receptor family and was reported to be induced by the Epstein-Barr virus (37) and overexpressed in different human cancers, such as non-small cell lung and (38) gastric cancer (39), and esophageal squamous cell carcinoma (40). In the present study, we found that CCR7 expression was upregulated in cells transfected with miR-20a-5p mimics and downregulated in cells transfected with miR-20a-5p inhibitor; thus, we speculate that reduced expression TNFRF 21 by miR-20a-5p could be responsible for CCR7 upregulation in HNSCC cells. Our research group has focused on studying the impact of CCR7 on HNSCC, and a previous study revealed that CCR7 was able to promote HNSCC cell proliferation, migration and invasion by activating NF- $\mathrm{NB}(21)$. Other researchers have reported that $\mathrm{NF}-\kappa \mathrm{B}$ can directly or indirectly regulate TNFRSF21 expression to protect the survival signaling from TNFRSF21-induced cell apoptosis (41). If this is true, our present study may have linked these molecules in HNSCC cells, e.g., CCR7 can upregulate the expression of NF- $\kappa \mathrm{B}$ and in turn activate TNFRSF21. In our present study, we found that upregulated miR-20a-5p expression inhibited TNFRSF21 expression, but upregulated CCR7 expression. We also found that miR-20a-5p was able to directly target TNFRSF21 to inhibit TNFRSF21 expression in PCI-37B cells. However, we are not sure whether there is a negative feedback loop among TNFRSF21, CCR7, and NF- $\kappa \mathrm{B}$ and how TNFRSF21 downregulated CCR7 expression in PCI-37B cells, thus we will undertake further investigation in our future study.

Our present study is just a proof-of-principle and does have a number of limitations. For example, we only used a single HNSCC cell line for gene knock-in and knockdown, which is not an appropriate research design. However, PCI-37A possesses a stronger adhesive property and has a poor gene transfection efficiency. Our future studies will acquire additional HNSCC cells for validation of our present data. Furthermore, we did not provide mechanistic data for how TNFRSF21 regulates CCR-7 expression in HNSCC cells. We should have performed a gene knockout experiment to assess the effect of TNFRSF21 on the regulation of CCR7 expression and altered HNSCC cell proliferation, migration and invasion in vitro. In conclusion, our present study revealed the oncogenic effect of miR-20a-5p on the promotion of HNSCC cell proliferation, migration, and invasion by downregulation of TNFRSF21. Further research is warranted to elucidate the precise mechanisms underlying the role of miR-20a-5p in HNSCC; for example, we will carefully select HNSCC cells with high or low expression of miR20a-5p to assess the effects of miR20a-5p knockdown and knock-in, respectively on the regulation of HNSCC phenotypes and gene targets. We will also perform in vivo experiments to assess the effects of miR-20a-5p on the regulation of tumor formation and growth in a nude mouse xenograft model of HNSCC cells. In addition, further research will reveal the underlying mechanism of miR-20a-5p upregulation in HNSCC to provide a molecular basis for a better understanding of HNSCC development.

\section{Acknowledgements}

The authors would like to thank the staff in The Central Laboratories, School of Stomatology, China Medical University for their technical assistance.

\section{Funding}

The present study was supported in part by grants from the National Natural Science Foundation of China (no. 81372877), 
the National Young Scholars Science Foundation of China (no. 81102058), the Public Welfare Fund Project for Science of Liaoning Province (no. 2011002001), the Excellent Talent Fund Project of Higher Education of Liaoning Province (no. LJQ2014087) and the Doctoral Scientific Research Foundation of Liaoning Province (no. 201501002).

\section{Availability of data and materials}

The datasets used during the present study are available from the corresponding author upon reasonable request.

\section{Authors' contributions}

CFS and FYL designed and conceived the study. HW, PP, MDL, SW and SJ performed the experiments. HW completed most of the experimental work and prepared the manuscript. CFS and FYL reviewed and edited the manuscript. All authors read and approved the final version of this manuscript and ensured the accuracy or integrity of any part of this work.

\section{Ethics approval and consent to participate}

All experimental protocols were approved by the Ethics Committee of China Medical University.

\section{Patient consent for publication}

Not applicable.

\section{Competing interests}

The authors declare that they have no competing interests.

\section{References}

1. Leemans CR, Braakhuis BJ and Brakenhoff RH: The molecular biology of head and neck cancer. Nat Rev Cancer 11: 9-22, 2011.

2. Grégoire V, Lefebvre JL, Licitra L and Felip E; EHNS-ESMO-ESTRO Guidelines Working Group: Squamous cell carcinoma of the head and neck: EHNS-ESMO-ESTRO Clinical Practice Guidelines for diagnosis, treatment and follow-up. Ann Oncol 21 (Suppl 5): v184-v186, 2010.

3. Bernstein E, Caudy AA, Hammond SM and Hannon GJ: Role for a bidentate ribonuclease in the initiation step of RNA interference. Nature 409: 363-366, 2001.

4. Zeng Y and Cullen BR: Sequence requirements for micro RNA processing and function in human cells. RNA 9: 112-123, 2003.

5. Arantes LM, Laus AC, Melendez ME, de Carvalho AC, Sorroche BP, De Marchi PR, Evangelista AF, Scapulatempo-Neto C, de Souza Viana L and Carvalho AL: MiR-21 as prognostic biomarker in head and neck squamous cell carcinoma patients undergoing an organ preservation protocol. Oncotarget 8: 9911-9921, 2017.

6. Sousa LO, Sobral LM, Matsumoto CS, Saggioro FP, López RV, Panepucci RA, Curti C, Silva WA Jr, Greene LJ and Leopoldino AM: Lymph node or perineural invasion is associated with low miR-15a, miR-34c and miR-199b levels in head and neck squamous cell carcinoma. BBA Clin 6: 159-164, 2016.

7. Hudcova K, Raudenska M, Gumulec J, Binkova H, Horakova Z, Kostrica R, Babula P, Adam V and Masarik M: Expression profiles of miR-29c, miR-200b and miR-375 in tumour and tumour-adjacent tissues of head and neck cancers. Tumour Biol 37: 12627-12633, 2016.

8. Koshizuka K, Hanazawa T, Fukumoto I, Kikkawa N, Okamoto Y and Seki N: The microRNA signatures: Aberrantly expressed microRNAs in head and neck squamous cell carcinoma. J Hum Genet 62: 3-13, 2017.
9. de Carvalho AC, Scapulatempo-Neto C, Maia DC, Evangelista AF, Morini MA, Carvalho AL and Vettore AL: Accuracy of microRNAs as markers for the detection of neck lymph node metastases in patients with head and neck squamous cell carcinoma. BMC Med 13: 108, 2015.

10. Hou B, Ishinaga H, Midorikawa K, Shah SA, Nakamura S, Hiraku Y, Oikawa S, Murata M and Takeuchi K: Circulating microRNAs as novel prognosis biomarkers for head and neck squamous cell carcinoma. Cancer Biol Ther 16: 1042-1046, 2015.

11. Hui AB, Lenarduzzi M, Krushel T, Waldron L, Pintilie M, Shi W, Perez-Ordonez B, Jurisica I, O'Sullivan B, Waldron J, et al: Comprehensive MicroRNA profiling for head and neck squamous cell carcinomas. Clin Cancer Res 16: 1129-1139, 2010.

12. Ramdas L, Giri U, Ashorn CL, Coombes KR, El-Naggar A, Ang KK and Story MD: miRNA expression profiles in head and neck squamous cell carcinoma and adjacent normal tissue. Head Neck 31: 642-654, 2009

13. Rahimy E, Kuo SZ and Ongkeko WM: Evaluation of non-coding RNAs as potential targets in head and neck squamous cell carcinoma cancer stem cells. Curr Drug Targets 15: 1247-1260, 2014.

14. Huang D, Bian G, Pan Y, Han X, Sun Y, Wang Y, Shen G, Cheng M, Fang $\mathrm{X}$ and $\mathrm{Hu}$ S: MiR-20a-5p promotes radio-resistance by targeting Rab27B in nasopharyngeal cancer cells. Cancer Cell Int 17: 32, 2017.

15. Chen Y, Wang X, Cheng J, Wang Z, Jiang T, Hou N, Liu N, Song $T$ and Huang C: MicroRNA-20a-5p targets RUNX3 to regulate proliferation and migration of human hepatocellular cancer cells. Oncol Rep 36: 3379-3386, 2016.

16. Cheng D, Zhao S, Tang H, Zhang D, Sun H, Yu F, Jiang W, Yue B, Wang J, Zhang M, et al: MicroRNA-20a-5p promotes colorectal cancer invasion and metastasis by downregulating Smad4. Oncotarget 7: 45199-45213, 2016.

17. Yang L, Liu F, Xu Z, Guo N, Zheng X and Sun C: Chemokine receptor 7 via proline-rich tyrosine kinase-2 upregulates the chemotaxis and migration ability of squamous cell carcinoma of the head and neck. Oncol Rep 28: 1659-1664, 2012.

18. Wang J, Zhang X, Thomas SM, Grandis JR, Wells A, Chen ZG and Ferris RL: Chemokine receptor 7 activates phosphoinositide-3 kinase-mediated invasive and prosurvival pathways in head and neck cancer cells independent of EGFR. Oncogene 24: 5897-5904, 2005.

19. Li P, Liu F, Sun L, Zhao Z, Ding X, Shang D, Xu Z and Sun C: Chemokine receptor 7 promotes cell migration and adhesion in metastatic squamous cell carcinoma of the head and neck by activating integrin alphavbeta3. Int J Mol Med 27: 679-687, 2011.

20. Zhao ZJ, Liu FY, Li P, Ding X, Zong ZH and Sun CF: CCL19-induced chemokine receptor 7 activates the phosphoinositide-3 kinase-mediated invasive pathway through Cdc42 in metastatic squamous cell carcinoma of the head and neck. Oncol Rep 25: 729-737, 2011.

21. Liu FY, Zhao ZJ, Li P, Ding X, Guo N, Yang LL, Zong ZH and Sun CF: NF- $\kappa B$ participates in chemokine receptor 7-mediated cell survival in metastatic squamous cell carcinoma of the head and neck. Oncol Rep 25: 383-391, 2011.

22. Fan X, Liu Y, Jiang J, Ma Z, Wu H, Liu T, Liu M, Li X and Tang H: miR-20a promotes proliferation and invasion by targeting APP in human ovarian cancer cells. Acta Biochim Biophys Sin 42: 318-324, 2010

23. Kang HW, Wang F, Wei Q, Zhao YF, Liu M, Li X and Tang $\mathrm{H}$ : miR-20a promotes migration and invasion by regulating TNKS2 in human cervical cancer cells. FEBS Lett 586: 897-904, 2012

24. Li X, Zhang Z, Yu M, Li L, Du G, Xiao W and Yang H: Involvement of miR-20a in promoting gastric cancer progression by targeting early growth response 2 (EGR2). Int J Mol Sci 14: 16226-16239, 2013

25. Zhou J, Liu R, Luo C, Zhou X, Xia K, Chen X, Zhou M, Zou Q, Cao P and Cao K: MiR-20a inhibits cutaneous squamous cell carcinoma metastasis and proliferation by directly targeting LIMK1. Cancer Biol Ther 15: 1340-1349, 2014

26. Xiong Y, Zhang L and Kebebew E: MiR-20a is upregulated in anaplastic thyroid cancer and targets $L I M K 1$. PLoS One 9: e96103, 2014.

27. Chang CC, Yang YJ, Li YJ, Chen ST, Lin BR, Wu TS, Lin SK, Kuo MY and Tan CT: MicroRNA-17/20a functions to inhibit cell migration and can be used a prognostic marker in oral squamous cell carcinoma. Oral Oncol 49: 923-931, 2013.

28. Hu J, Ge W and Xu J: HPV 16 E7 inhibits OSCC cell proliferation, invasion, and metastasis by upregulating the expression of miR-20a. Tumour Biol 37: 9433-9440, 2016. 
29. Yu Z, Willmarth NE, Zhou J, Katiyar S, Wang M, Liu Y McCue PA, Quong AA, Lisanti MP and Pestell RG: microRNA 17/20 inhibits cellular invasion and tumor metastasis in breast cancer by heterotypic signaling. Proc Natl Acad Sci USA 107: 8231-8236, 2010.

30. Singh B, Ronghe AM, Chatterjee A, Bhat NK and Bhat HK: MicroRNA-93 regulates NRF2 expression and is associated with breast carcinogenesis. Carcinogenesis 34: 1165-1172, 2013.

31. Liu S, Patel SH, Ginestier C, Ibarra I, Martin-Trevino R, Bai S, McDermott SP, Shang L, Ke J, Ou SJ, et al: MicroRNA93 regulates proliferation and differentiation of normal and malignant breast stem cells. PLoS Genet 8: e1002751, 2012.

32. Pan G, Bauer JH, Haridas V, Wang S, Liu D, Yu G, Vincenz C, Aggarwal BB, Ni J and Dixit VM: Identification and functional characterization of DR6, a novel death domain-containing TNF receptor. FEBS Lett 431: 351-356, 1998.

33. Sedý J, Bekiaris V and Ware CF: Tumor necrosis factor superfamily in innate immunity and inflammation. Cold Spring Harb Perspect Biol 7: a016279, 2014.

34. Pfeffer K: Biological functions of tumor necrosis factor cytokines and their receptors. Cytokine Growth Factor Rev 14: 185-191, 2003.

35. Thorburn A: Death receptor-induced cell killing. Cell Signal 16: 139-144, 2004
36. Tisch M, Kyrberg H, Weidauer H, Mytilineos J, Conradt C, Opelz G, Maier H: Human leukocyte antigens and prognosis in patients with head and neck cancer: Results of a prospective follow-up study. Laryngoscope 112: 651-657, 2002.

37. Sharma N, Benechet AP, Lefrancois L and Khanna KM: CD8 $\mathrm{T}$ cells enter the Splenic T cell zones independently of CCR7, but the subsequent expansion and trafficking patterns of effector $T$ cells after infection are dysregulated in the absence of CCR7 migratory cues. J Immunol 195: 5227-5236, 2015.

38. Takanami I: Overexpression of CCR7 mRNA in nonsmall cell lung cancer: Correlation with lymph node metastasis. Int J Cancer 105: 186-189, 2003.

39. Mashino K, Sadanaga N, Yamaguchi H, Tanaka F, Ohta M, Shibuta $\mathrm{K}$, Inoue $\mathrm{H}$ and Mori $\mathrm{M}$ : Expression of chemokine receptor CCR7 is associated with lymph node metastasis of gastric carcinoma. Cancer Res 62: 2937-2941, 2002.

40. Ding Y, Shimada Y, Maeda M, Kawabe A, Kaganoi J, Komoto I, Hashimoto $\mathrm{Y}$, Miyake $\mathrm{M}$, Hashida $\mathrm{H}$ and Imamura $\mathrm{M}$ : Association of CC chemokine receptor 7 with lymph node metastasis of esophageal squamous cell carcinoma. Clin Cancer Res 9: 3406-3412, 2003.

41. Kasof GM, Lu JJ, Liu D, Speer B, Mongan KN, Gomes BC and Lorenzi MV: Tumor necrosis factor-alpha induces the expression of DR6, a member of the TNF receptor family, through activation of NF-kappaB. Oncogene 20: 7965-7975, 2001. 